\title{
Common Coupling and Pointer Variables, with Application to a Linux Case Study
}

\author{
Stephen R. Schach Tokunbo O. S. Adeshiyan Daniel Balasubramanian \\ Gabor Madl Esteban P. Osses Sameer Singh \\ Karlkim Suwanmongkol Minhui Xie Dror G. Feitelson* \\ Department of Electrical Engineering and Computer Science \\ Vanderbilt University, Nashville, TN 37235
}

June 29, 2005

\begin{abstract}
Both common coupling and pointer variables can exert a deleterious effect on the quality of software. The situation is exacerbated when pointer variables are assigned to global variables, that is, when an alias to a global variable is created. When this occurs, the number of global variables increases, and it becomes considerably harder to compute quality metrics correctly. However, unless aliasing is taken into account, variables may incorrectly appear to be unreferenced (neither defined nor used), or to be used without being defined. These ideas are illustrated by means of a case study of common coupling in the Linux kernel.
\end{abstract}

*On sabbatical leave from Hebrew University 
Key words: Common coupling, aliasing, pointer variables, Linux, global variables, definition-use analysis.

\section{Introduction}

The goal of software engineering is to produce high-quality maintainable software. But there is little agreement regarding how quality and maintainability should be measured, and whether they can be measured directly. Over the years, various indirect measures have therefore been proposed. The degree of common coupling is one of them: Significant common coupling is considered bad, so low levels of common coupling are taken to indicate high quality and maintainability [Schach, 2005]. Such metrics are especially useful for the comparison of contending software development practices, such as open-source vs. closed source.

Common coupling refers to the use of global variables. Using global variables is bad because it violates the principles of encapsulation, information hiding, and abstraction. Global variables are volatile, in the sense that their value may be changed in unpredictable ways, due to side effects of called functions.

Using global variables is bad practice; allowing pointer variables to point to global variables is even worse. When global variables are used directly, it is relatively straightforward to find all instances of these global variables and check their effect. But with pointer variables, a global variable may have several aliases. This makes it impractical to track the possible interactions among different modules, and increases the risk of undesirable effects.

We demonstrate the problems that stem from using pointers to global variables by means of a case study of common coupling in the Linux kernel.

The remainder of this paper is organized as follows: In Section 2 we discuss cou- 
pling issues, especially global variables and common coupling. The effects of pointer variables on common coupling are described in Section 3. The Linux case study is presented in Section 4, and the results of the case study in Section 5. Our conclusions appear in Section 6.

\section{Coupling of Software Modules}

Successful software projects are those that meet their specifications within predefined budget and time constraints. This metric is applicable to traditional closed-source software development, and has been measured for many thousands of projects. In fact, such measurements are the basis for the claim that the software industry is in a crisis; studies routinely show that the majority of projects fail to meet their targets [Jones, 1995, Johnson et. al., 2001].

Regrettably, this straightforward metric cannot be applied to open-source software projects, because they typically have no detailed specifications, no budget, and no deadlines. Therefore, indirect metrics have to be found. Given the availability of the source code, it is natural to consider metrics that are based on the code itself, that is, metrics for code quality. These have the additional appeal of being quantitative, objective, and amenable to mechanized evaluation.

One such metric is the degree of coupling found in the code. Coupling between software modules measures the degree to which they are dependent on each other. One of the basic tenets of software engineering is that modules should be only weakly coupled together, because this promotes easier maintenance and reuse [Schach, 2005, Offutt et. al., 1993]; contrariwise, strong coupling makes modules harder to understand and increases the propensity for errors [Binkley and Schach, 1998, Rilling and Klemola, 2003].

There are many different types of coupling that can occur between software mod- 
ules [Offutt et. al., 1993]. Coupling means that one module depends on the other, typically in the form of using data that are produced by the other module. The distinctions are based on whether passed data are also used for control or not, and whether they are passed uni-directionally or bi-directionally.

Some form of coupling is obviously needed in order to allow the modules to work together as parts of a single application. But not all forms of coupling are equal. In particular, it is widely agreed that so called "common coupling" should be avoided. This refers to the use of global (shared) variables, harking back to the COMMON keyword from FORTRAN.

Global variables are bad mainly because they allow for side effects. Consider a module that uses a global variable $g$, and calls a function $f()$ from another module. When the function returns, the value of g may have changed. Moreover, future changes to $f()$ may cause new and unexpected behavior of g. Consequently, the programmer cannot rely on g remaining consistent, and needs to handle it with extreme care. Another reason that common coupling is considered bad is that it is susceptible to clandestine increase, where the coupling of a given module increases without the module itself being modified in any way, just because other modules have been modified [Schach et. al., 2003].

\section{The Effect of Pointer Variables on Common Cou- pling}

Programming languages such as $\mathrm{C}$ allow for pointer variables that point to other variables. This mechanism can be used to create aliases; a given variable can be accessed using its original name, and also through pointer variables that point to it. 
Although pointer variables have some important applications in dynamic data structures, their indiscriminate use causes many problems. Because these pointers are variables, they can be assigned at runtime. Accordingly, a pointer may point to different variables at different times in the execution of the program. This makes it extremely hard, or even impossible, to perform a static analysis of the behavior of the program.

One alternative is to utilize conservative approaches. For example, all modules that access a global data structure in some way will be considered to be common coupled, even if there are actually subsets of modules that do not really depend on each other. For example, a global data structure may consist of two fields, $x 1$ and $x 2$. One set of modules may access only $x 1$ and another set may access only $x 2$. In such a case, claiming that the modules of the two sets are common coupled is inaccurate.

In other words, in order to determine the extent of common coupling (and, hence, measure program quality indirectly), we need to be able to identify every instance of a global variable. The presence of pointer variables can make such an determination difficult or even impossible.

We now illustrate these ideas with a case study.

\section{Case Study: The Linux Kernel}

Tabulating the degree of common coupling has been used to assess the quality of the code in the Linux operating system kernel, and how it changes with time. The original study by Schach et al. found that the number of instances of common coupling grows exponentially with version number [Schach et. al., 2002]. A follow-up by Yu et al. found that much of the common coupling was of an especially bad type that coupled kernel modules to nonkernel modules [Yu et. al., 2004]. 


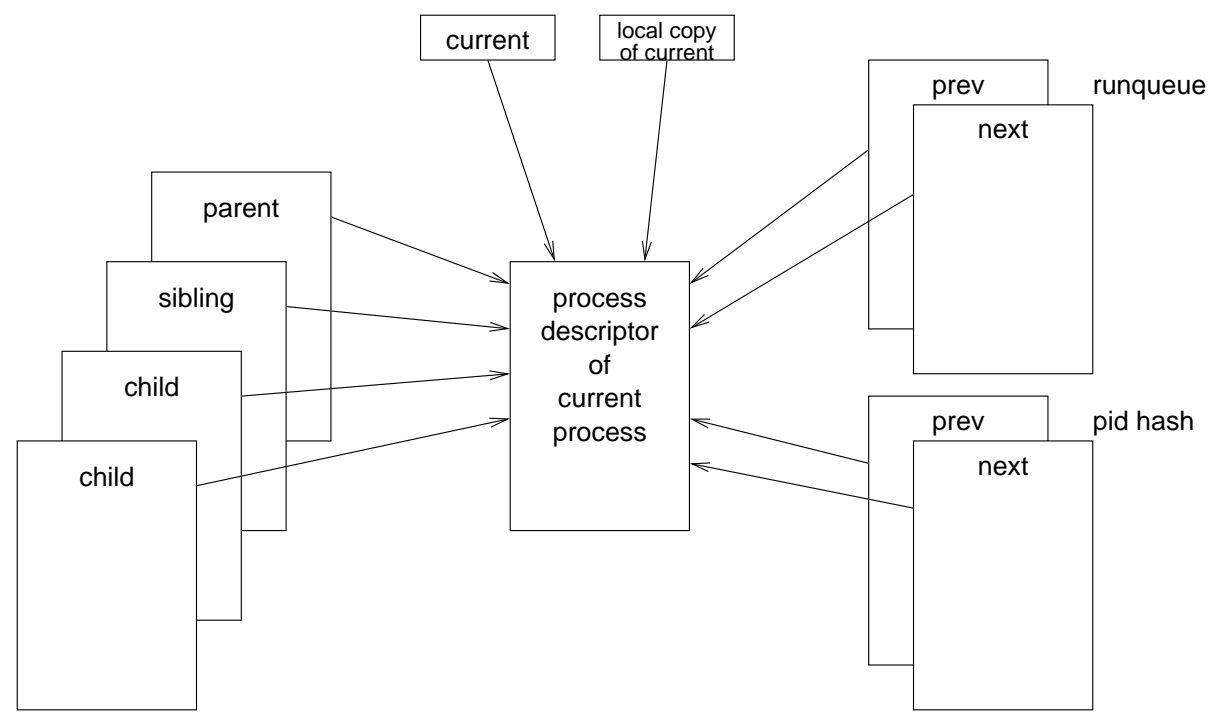

Figure 1: Pointers that can be used to access a process descriptor in Linux.

A deficiency of these studies is that they were based on a lexical analysis of the Linux source code. In other words, they identified references to global variables only if the same name was used. However, the Linux kernel is rife with instances of references to global variables using pointer variables. To see how presence of these aliases affected the accuracy of these studies, consider the most heavily used global variables in Linux, which are the process descriptors.

Linux (like practically any other operating system) maintains a process descriptor for each process in the system. This data structure resides in an 8-KB block of memory that also holds the kernel stack of the process. This memory area is allocated dynamically when the process is created. Thus there is no pre-defined array of process descriptors, as there was in early versions of Unix.

In Linux, a process descriptor is a structure of type task_struct, which has 105 fields ${ }^{1}$. Process descriptors are most commonly accessed via global pointer variable current, which points to the currently running process. (In reality, it is implemented

\footnotetext{
${ }^{1}$ This and other references to the Linux source code refer to kernel version 2.4.20, in order to be able to draw comparisons with previous studies, especially [Yu et. al., 2004].
} 
as a mask on the stack pointer register, based on the fact that the kernel stack and process descriptor are co-located in the same memory block.) Of the 105 fields, 25 are pointers to various data structures; of these, 9 are pointers to other instances of type task_struct. They are used to link the process descriptor into three separate data structures: the run-queue (or some other list of processes), the pid (process identifier) hash table, and the tree of process family relationships (connecting processes to their parent, siblings, and children). The run-queue links in particular are used by the scheduler to traverse all the runnable processes in the system and select one for execution. To complicate matters further, there are at least 117 places in the code where current is copied to a local pointer that is then used to access the current process descriptor. In short, there are many different ways to access a process descriptor, all using pointers (Fig. 1). The earlier works on common coupling in Linux (e.g. [Yu et. al., 2004]) only considered the use of current itself.

A similar situation occurs for data structures that are pointed to by the process descriptor. Sixteen of the fields of task_struct are pointers to other structures, including ones that describe the process's memory layout and open files; some subfields are also pointers to other structures. All these are often accessed by double (or more levels of) indirection using current. But there are at least 148 cases where a local copy of a pointer is made, affecting the access to 28 different subfields (of a total of 249 subfields). There are also at least 24 cases of aliases to aliases (that is, a local pointer to current or to a field is copied to another local pointer). Again, previous studies counted only the accesses using current, and missed those that use a local copy. 


\begin{tabular}{lr}
\hline Referenced via current: & 89 Fields \\
Referenced only via aliasing: & 1 Fields \\
Never referenced: & 15 Fields \\
Total fields: & 105 Fields \\
\hline
\end{tabular}

Table 1: Fields of task_struct referenced via current.

\section{$5 \quad$ Results}

We investigated the common coupling induced by current. First we looked solely at the fields of task_struct ${ }^{2}$. Then we also considered the fields of the other types of structures pointed to by fields of task_struct. In each case, we considered the situation with and without aliasing.

\subsection{Analysis of Fields of task_struct}

In this subsection, we consider the fields of task_struct referenced using current. As shown in Table 1, when we consider just current itself and the fields to which it points, 89 of the 105 fields of task_struct are referenced. An example of such a reference (to field processor) is

$$
\text { current- }>\text { processor }=0 \text {; }
$$

If we also consider all aliases for current, a single additional field is referenced. These are the statement in question:

$$
\begin{aligned}
& \text { struct task_struct } * \text { tsk }=\text { current; } \\
& \text { tsk->vfork_done = NULL; }
\end{aligned}
$$

Clearly, aliasing has a negligible impact on the fields of task_struct accessed via current.

\footnotetext{
${ }^{2}$ In what follows, for brevity we use the informal terminology "fields of task_struct" rather than the more precise "fields of instances of type task_struct."
} 


\begin{tabular}{lr}
\hline Referenced via current: & 280 Subfields \\
Referenced only via aliasing: & 58 Subfields \\
Total referenced fields: & 338 Subfields \\
\hline
\end{tabular}

Table 2: Subfields of current referenced via current or via an alias.

\subsection{Wider Analysis}

We now consider all fields referenced, directly or indirectly, by current. For example, consider the statement

$$
\text { current- }>\text { fs- }>\text { altrootmnt }=\text { mnt; }
$$

Here, pointer variable current points to pointer field fs in task_struct. Pointer fs is a pointer to a structure of type fs_struct. Field altrootmnt is a field of a structure of type fs_struct and is set equal to mnt. In this case, the fields referenced are fs and altrootmnt; we refer to such fields collectively as subfields of current.

Another example is

$$
\text { sig = fpu_emulator_cop1Handler(0, regs, \&current->thread.fpu.soft); }
$$

In this example, current points to a struct of type task_struct, which contains a field thread, a struct of type thread_struct. The latter has a field fpu which is a struct of type fp_status; this struct has a field soft. Here the subfields of current are thread, fpu, and soft.

Table 2 shows the results when all subfields referenced using current and its aliases are considered. The results in Table 2 incorporate those of Table 1 . Now there are 58 fields that are accessed only via aliases. In other words, over 17 percent of the 338 fields would not be taken into account if aliasing were ignored. 


\subsection{Unreferenced and Undefined Global Variables}

Every instance of a variable in a program is either a definition of that variable (that is, a change to the value of that variable) or a use of that variable (that is, a utilization of the current value of that variable).

An unreferenced field is one that is neither defined nor used in the statements we examined. As shown in Tables 3 and 2, following aliases exposes 58 additional subfields that are not seen when only references using current are considered. They are therefore classified as unreferenced when aliasing is not considered. But even with alising there are 6 unreferenced fields. These 6 fields fall into the following categories:

1. "Fields" that were found by mistake, due to outdated comments that mention fields that no longer exist. There were 4 such fields.

2. A field (thread.usp) that exists, is mentioned in a comment, but is not referenced from current. However, it could be referenced via some other mechanism of which we are unaware.

3. A field (thread.esp) that occurs in only an assembler statement. We have set this instance aside until we have done the necessary research into the nature of common coupling between a second-generation language (assembler) and a third-generation language $(\mathrm{C})$.

An undefined field is one that is used but not defined in the statements we examined. As previously mentioned, without aliasing there were 64 unreferenced fields. Aliasing caused 22 of them to become undefined, and 36 to become defined. Also, without aliasing there were 78 undefined fields. Aliasing caused 11 of them to become defined. So, as shown in Table 3, the number of unreferenced fields dropped from 64 to 6 , and the number of undefined fields increased from 78 to $89(=78+22-11)$. 


\begin{tabular}{lcccc}
\hline & Unreferenced & Undefined & Defined & Total fields referenced \\
\hline Without aliasing: & 64 & 78 & 202 & 278 \\
With aliasing: & 6 & 89 & 249 & 338 \\
\hline
\end{tabular}

Table 3: The effect of following aliases.

\begin{tabular}{lrr}
\hline Category 0: & 118 subfields & $(58.4 \%)$ \\
Category 1: & 5 subfields & $(2.5 \%)$ \\
Category 2: & 27 subfields & $(13.4 \%)$ \\
Category 3: & 0 subfields & $(0.0 \%)$ \\
Category 4: & 7 subfields & $(3.5 \%)$ \\
Category 5: & 45 subfields & $(22.3 \%)$ \\
Total: & 202 subfields & $(100.0 \%)$ \\
\hline
\end{tabular}

Table 4: Results of categorizing subfields of current without any aliasing.

\subsection{Categorization of Common Coupling}

Yu et al. [Yu et. al., 2004] categorized common coupling in kernel-based software. In brief, $\mathrm{Yu}$ et al. set up five categories of common coupling on the basis of definitionuse analysis (for full details, the reader should refer to [Yu et. al., 2004]). Subsequently, Feitelson et al. added a sixth category; the interested reader should consult [Feitelson et. al., 2005] for details. For the purposes of this paper, however, all that is needed is the knowledge that there exists a six-way categorization of common coupling in kernel-based software on the basis of definition-use analysis, and that the higher the category number, the more undesirable.

Without aliasing, 202 fields of current are defined, as shown in Table 3. The

\begin{tabular}{lrr}
\hline Category 0: & 154 subfields & $(61.8 \%)$ \\
Category 1: & 5 subfields & $(2.0 \%)$ \\
Category 2: & 27 subfields & $(10.8 \%)$ \\
Category 3: & 3 subfields & $(1.2 \%)$ \\
Category 4: & 7 subfields & $(2.8 \%)$ \\
Category 5: & 53 subfields & $(21.3 \%)$ \\
Total: & 249 subfields & $(100.0 \%)$ \\
\hline
\end{tabular}

Table 5: Results of categorizing subfields of current incorporating aliasing. 


\begin{tabular}{crrrrrrrr}
\hline & \multicolumn{10}{c}{ to } \\
\cline { 2 - 9 } from & UR & UD & 0 & 1 & 2 & 3 & 4 & 5 \\
\hline Unreferenced: & 6 & 22 & 33 & 0 & 3 & 0 & 0 & 0 \\
Undefined: & 0 & 67 & 9 & 0 & 0 & 1 & 0 & 1 \\
Category 0: & 0 & 0 & 112 & 0 & 0 & 0 & 2 & 4 \\
Category 1: & 0 & 0 & 0 & 5 & 0 & 0 & 0 & 0 \\
Category 2: & 0 & 0 & 0 & 0 & 24 & 2 & 0 & 1 \\
Category 3: & 0 & 0 & 0 & 0 & 0 & 0 & 0 & 0 \\
Category 4: & 0 & 0 & 0 & 0 & 0 & 0 & 5 & 2 \\
Category 5: & 0 & 0 & 0 & 0 & 0 & 0 & 0 & 45 \\
\hline
\end{tabular}

Table 6: Re-categorization of fields of current as a result of considering references made via aliases.

categorization of these global variables is shown in Table 4. When aliasing is taken into account, the number of defined fields increases to 249; their distribution is shown in Table 5.

We observe first that the number of fields increases by nearly 25 percent when aliasing is taken into account, and second that the distribution of global variables among the categories changes. In particular, some fields moved "up" to higher categories, which in general indicates a stronger form of coupling, which is undesirable. Notably, in the initial categorization there were no fields in category 3 , but with aliasing three such fields were found. More significantly, the number of fields in highly undesirable category 5 increased from 45 to 53 , an increase of over 17 percent. These changes are shown in more detail in Table 6.

\subsection{References to Global Variables}

Finally, we counted the number of references to each of the global variables accessible from current. Comparing Tables 7 and 8, we see that not only does the number of references increase when aliasing is taken into account, the percentage of references to different categories changes as well. 


\begin{tabular}{|c|c|c|}
\hline & Kernel & Non-kernel \\
\hline Category 0: & $0.0 \%)$ & $1410(23.3 \%)$ \\
\hline Category 1: & $1.4 \%)$ & $0.3 \%)$ \\
\hline Category 2: & $96(21.8 \%)$ & $3.2 \%)$ \\
\hline Category 3: & $0(0.0 \%)$ & $0.0 \%)$ \\
\hline Category 4: & $4.1 \%)$ & $2.2 \%)$ \\
\hline Category 5: & $320(72.7 \%)$ & $4310(71.1 \%)$ \\
\hline Total: & $440(100.0 \%)$ & $6060(100.0 \%)$ \\
\hline
\end{tabular}

Table 7: Results of analyzing individual occurrences of fields of current without aliasing.

\begin{tabular}{|c|c|c|}
\hline & Kernel & Non-kernel \\
\hline Category 0: & $0.0 \%)$ & $1894(25.3 \%)$ \\
\hline Category 1: & $1.0 \%)$ & $0.2 \%)$ \\
\hline Category 2: & $96(15.2 \%)$ & $2.6 \%)$ \\
\hline Category 3: & $2.1 \%)$ & $0.1 \%)$ \\
\hline Category 4: & $2.5 \%)$ & $2.2 \%)$ \\
\hline Category 5: & $500(79.2 \%)$ & $5214(69.5 \%)$ \\
\hline Total: & $631(100.0 \%)$ & $7499(100.0 \%)$ \\
\hline
\end{tabular}

Table 8: Results of analyzing individual occurrences of fields of current with aliasing.

\subsection{Threats to the Validity of the Linux Case Study}

In this case study, we have considered all fields referenced directly or indirectly by pointer variable current. We have also considered aliases of current and of pointer variables referenced directly or indirectly by current. However, we have not considered all of the many aliases in Linux. A consequence is that there may be many more global variables than those that we have identified.

We have identified a number of fields that apparently either are never referenced, or are never defined. Some Linux fields are initialized by copying. That is, sometimes a structure is created as a copy of a preinitialized "standard" version of the structure. The structure as a whole is copied, thereby defining the relevant fields. In other words, the only definition mechanism we have considered is assignment. Accordingly, 
we may have miscategorized some global variables as unreferenced.

\section{Conclusions}

It is widely agreed that common coupling, that is, the use of global variables, should be minimized, and that pointer variables need to be handled with care. In this paper we have demonstrated four examples of what can happen when pointer variables and common coupling interact.

First, the creation of an alias for a global variable means another global variable has been created. That is, aliasing of global variables is antithetical to the goal of minimizing the number of global variables in a program. This holds irrespective of whether the global variable in question is a pointer variable (as is the case in this paper). However, the severity of the situation is aggravated when the global variable in question is a pointer. When a pointer to a structure is a global variable, then all the fields of that structure become global variables, too. Creating an alias to the pointer results in even more global variables. That is, aliasing can create global variables that do not exist in the absence of aliasing.

Second, if fields of the structure in question are themselves pointer variables, then the items to which they point are also global variables, and this may be taken to any level. Again, if there is aliasing as well, then the number of global variables can be further increased.

Third, the presence of aliasing means that considerable additional work may have to be done in order to compute quality metrics correctly. These metrics include the number of global variables and their categorization in terms of definition-use analysis.

Fourth, without taking aliasing in account, variables may incorrectly appear to be unreferenced (neither defined nor used), or to be used without being defined. 
In conclusion, the combination of global variables and pointer variables is highly undesirable. In those situations where global variables are essentially unavoidable, pointer variables should be eschewed.

\section{References}

[Binkley and Schach, 1998] Binkley A. B. and Schach S. R., "Validation of the coupling dependency metric as a predictor of run-time failures and maintenance measures". In 20th Intl. Conf. Softw. Eng., pp. 452-455.

[Feitelson et. al., 2005] Feitelson D. G., Adeshiyan T. O. S., Balasubramanian D., Etsion Y., Madl G., Osses E. P., Singh S., Suwanmongkol K., Xie C., and Schach S. R., Fine-Grain Analysis of Common Coupling and its Application to a Linux Case Study. Technical Report 2005-37, Hebrew University School of Computer Science and Engineering.

[Johnson et. al., 2001] Johnson J., Boucher K. D., Connors K., and Robinson J., "Project management: the criteria for success". Softwaremag.com. URL www.softwaremag.com/archive/2001feb/CollaborativeMgt.html.

[Jones, 1995] Jones C., Patterns of Software System Failure and Success. Intl Thomson Computer Pr $(\mathrm{Sd})$.

[Offutt et. al., 1993] Offutt A. J., Harrold M. J., and Kolte P., "A software metric system for module coupling". J. Syst. ES Softw. 20(3), pp. 295-308.

[Rilling and Klemola, 2003] Rilling J. and Klemola T., "Identifying comprehension bottlenecks using program slicing and cognitive complexity metrics". In 11th IEEE Intl. Workshop Program Comprehension, pp. 115-124. 
[Schach, 2005] Schach S. R., Object-Oriented and Classical Software Engineering. McGraw-Hill, 6 ed.

[Schach et. al., 2002] Schach S. R., Jin B., Wright D. R., Heller G. Z., and Offutt A. J., "Maintainability of the Linux kernel". IEE Proc.-Softw. 149(2), pp. $18-23$.

[Schach et. al., 2003] Schach S. R., Jin B., Wright D. R., Heller G. Z., and Offutt J., "Quality impacts of clandestine common coupling". Softw. Quality J. 11, pp. 211-218.

[Yu et. al., 2004] Yu L., Schach S. R., Chen K., and Offutt J., "Categorization of common coupling and its application to the maintainability of the Linux kernel". IEEE Trans. Softw. Eng. 30(10), pp. 694-706. 